\title{
Test Site Location
}

National Cancer Institute

\section{Source}

National Cancer Institute. Test Site Location. NCI Thesaurus. Code C90471.

The geographic location in which a test is managed. 